\title{
Crystallization and rheological behavior of POSS filled polypropylene
}

Zhiyong Zhou, ${ }^{1}$ Chunfa Ouyang, ${ }^{2}$ Yong Zhang, ${ }^{1}$ Yinxi Zhang, ${ }^{1 *}$ Nianwei Yin ${ }^{1}$

${ }^{1}$ Research Institute of Polymer Materials, School of Chemistry and Chemical Technology, Shanghai Jiao Tong University, Shanghai 200240, China; fax: +86(021)-54741297; tel: +86-(021)-54742671; e-mail: yxzhang@sjtu.edu.cn

2Department of Materials Science and Engineering, Shanghai Institute of Technology, Shanghai, 200235, China

(Received: 23 May, 2008; published 06 April, 2009)

\begin{abstract}
Octaaminophenyl polyhedral oligomeric silsesquioxane (OapPOSS) was prepared as nanofillers and filled to isotactic polypropylene (PP). Maleic anhydridegrafted PP (MAPP) was selected as compatibilizer. The morphology, crystallization, melting behavior and rheologcial behavior of PP/OapPOSS composites with or without MAPP were studied by scanning electron microscopy, differential scanning calorimetry, polarized optical microscope, oscillatory and capillary rheological analysis. The results showed that the morphology, crystallization and rheological behavior of the composites were strongly influenced by the existence of MAPP. MAPP improves the compatibility between PP and OapPOSS because of the reaction of OapPOSS with MAPP. The OapPOSS retards the crystallization of PP while promoting it when MAPP is added. MAPP could decrease the viscosity of PP/OapPOSS probably due to the weaker particleparticle interaction and interface slipping.
\end{abstract}

\section{Introduction}

In recent years, the use of polyhedral oligomeric silsesquioxane (POSS) nanoparticles (Figure 1) as modifiers of polymers have drawn great attention following the development of efficient synthetic protocols for the preparation of the inorganic $\mathrm{Si}_{8} \mathrm{O}_{12}$ core [1]. POSS can be effectively incorporated into polymers by copolymerization, grafting or blending.<smiles>[R][Si]12O[Si]3([R])O[Si]4([R])O[Si]([R])(O1)O[Si]1([R])O[Si]([R])(O2)O[Si]([R])(O3)O[Si]([R])(O4)O1</smiles>

Fig. 1. Structure of polyhedral oligomeric silsesquioxane. 
Up to date POSS and its derivatives have been reported as candidates in modifying a wide range of thermoplastics such as polyolefin [2-10], polystyrene [11], poly(methyl methacrylate) [12], polycarbonate [13], poly(ethylene terephthalate) [14, 15], and polyamide [15, 16] and a few thermoset systems [18-21]. The incorporation of POSS or its derivatives into polymers can lead to some dramatically improved properties, such as the increase in use temperature, oxidation resistance, surface hardening and improved mechanical properties, as well as reductions in flammability, heat evolution, and viscosity during processing, etc.[22]

However, most kinds of POSS are immiscible in general organic systems because of the weak specific interactions in these organic-inorganic hybrids. The physical properties of polymer/POSS composites are strongly influenced by the miscibility between the host polymer and the POSS moiety [23, 24]. Ni et al. [18] reported that epoxy resin containing nitrophenyl POSS was phase separated while aminophenyl POSS-containing hybrid was homogeneous. Zhao et al. [13] reported that trisilanol POSS molecules generally provided better compatibility with polycarbonate than POSS with fully-saturated cage structure, and phenyl-substituted POSS grades were shown to be more compatible with polycarbonate than fillers with other functional groups. Fina et al. [5] reported that the compatibility with POSS and polypropylene (PP) increased with increasing alkyl chain length from octamethyl POSS to octasiobutyl-POSS. POSS has been introduced into PP by copolymerization or physical blending to study the morphology, crystallization behavior, rheological behavior, mechanical properties etc [5-7, 10, 25-30]. The properties of POSS filled PP were strongly affected by the miscibility of POSS and PP. The compatibilizer or coupling agent has been widely used in polymer blends and polymer/filler composites to improve the compatibility in immiscible blends or polymer/filler composites. Up to now, there is little literature about the use of compatibilizer for polymer/POSS nanacomposites. Octaaminophenyl POSS (OapPOSS) appears to offer excellent potential as a nanoconstruction site for preparing materials ranging from high temperature nanocomposites, to precursors, organic light-emitting diodes, multiarmed stars and templates for high temperature porous materials for use in catalysis, sensing, separations, etc [21]. OapPOSS had also been filled in thermoset polymers $[18,31,32]$. In this article, OapPOSS was prepared and used as a nanofiller to fill isotactic PP and maleic anhydride-grafted PP (MAPP) was used as compatibilizer. As expected, OapPOSS with small particle size would disperse more uniformly in PP, and crystallization and rheological behaviors of this system were also changed when MAPP was added due to the reaction of MAPP with OapPOSS. This strategy provides a cheaper and less time-consuming method than the development of new POSS or copolymerization POSS with polymers.

\section{Results and discussion}

The SEM micrographs of PP composites were shown in Figure 2. For the PP/OapPOSS2 composites [Fig. 2(a)], OapPOSS dispersed as particles with the diameter changed from $50 \mathrm{~nm}$ to $1 \mu \mathrm{m}$, while the particle diameter of PP/MAPP/OapPOSS2 composites became smaller and changed from $50 \mathrm{~nm}$ to 100 $\mathrm{nm}$. The smaller particle size should be related to the presence of 10\% MAPP. It can be concluded that the MAPP improve the compatibility of PP and OapPOSS and promote a fine dispersion of OapPOSS due to the reaction of OapPOSS with the maleic anhydride. 

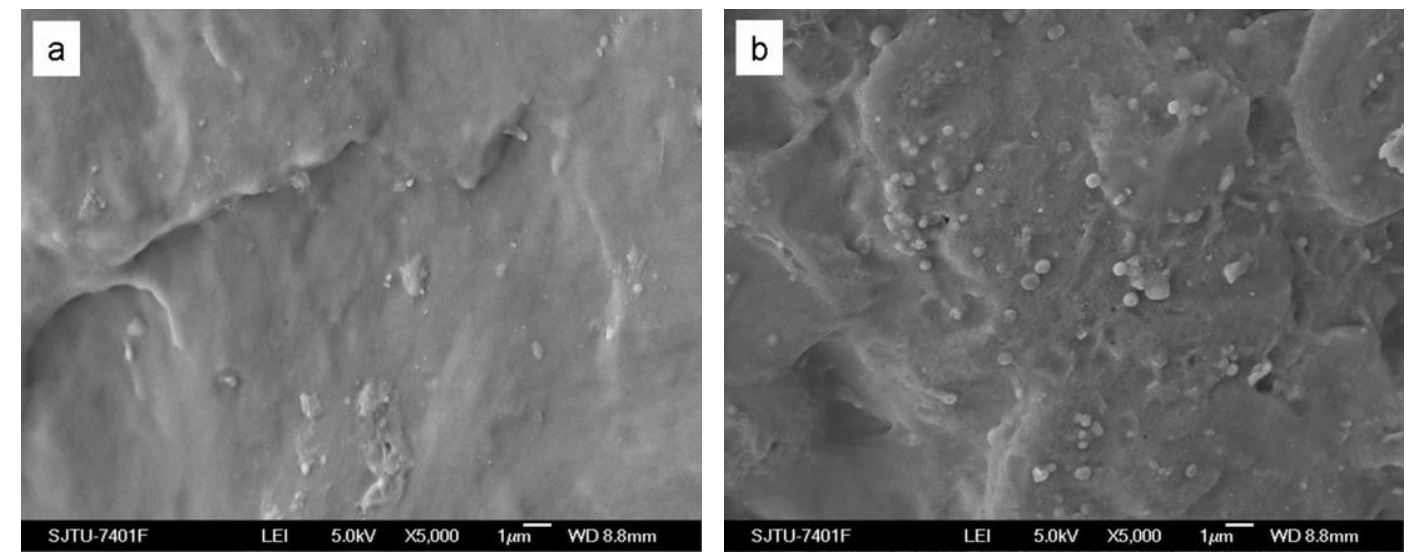

Fig. 2. SEM micrographs of PP composites: (a) PP/OapPOSS2 and (b) PP/MAPP/ OapPOSS2.

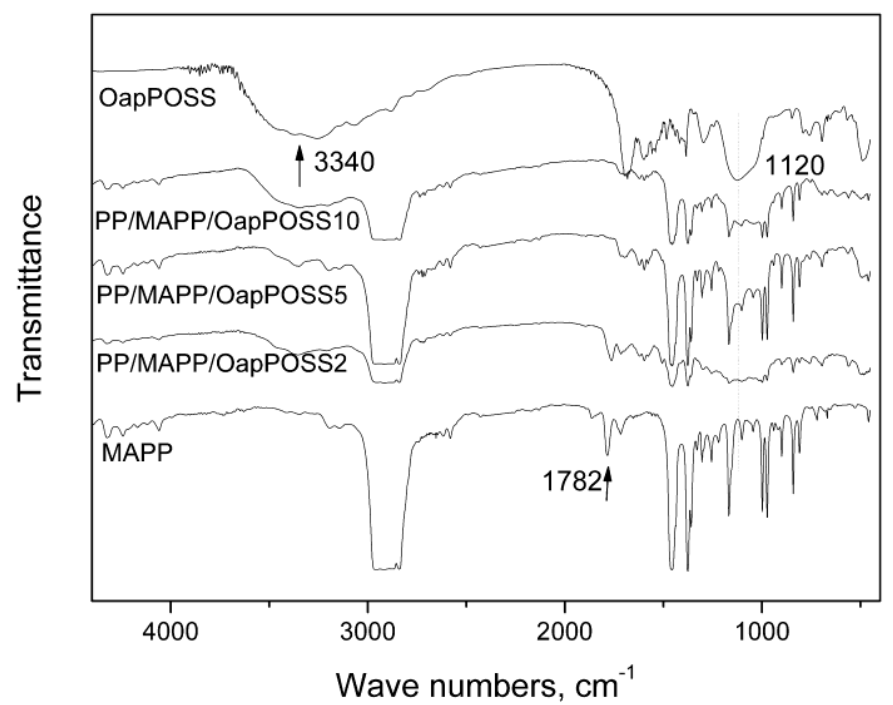

Fig. 3. The FTIR spectra of PP/MAPP/OapPOSS.

The FTIR spectra of PP/MAPP/OapPOSS composites after removing the unreactive OapPOSS are shown in Figure 3 . The stretching band of $\mathrm{C}=\mathrm{O}$ of maleic anhydride at $1782 \mathrm{~cm}^{-1}$ shiffed to lower wave number, at the same time, the characteristic peaks of OapPOSS (near 3340 and $1120 \mathrm{~cm}^{-1}, \mathrm{VN}-\mathrm{H}$ and $\mathrm{vSi}-\mathrm{O}-\mathrm{Si}$, respectively) increased with increasing OapPOSS content, indicating the reactive OapPOSS increased with increasing OapPOSS content. As such, the amine group of OapPOSS would react with the carbonyl groups of maleic anhydride of MAPP, as shown in Scheme 1.

Figures 4 and 5 show the DSC cooling and reheating melt thermograms of PP composites, respectively. The nonisothermal crystallization parameters according to the DSC thermograms are listed in Table 1. For PP/OapPOSS, the peak temperature, $T_{\mathrm{c}}$ and $T_{\text {onset }}$, which is the temperature at the intercept of the tangents at the baseline and the high-temperature side of the exothem, decreased with increasing OapPOSS content. While $T_{\mathrm{c}}$ and $T_{\text {onset }}$ of PP/MAPP/OapPOSS increased with increasing OapPOSS content. 


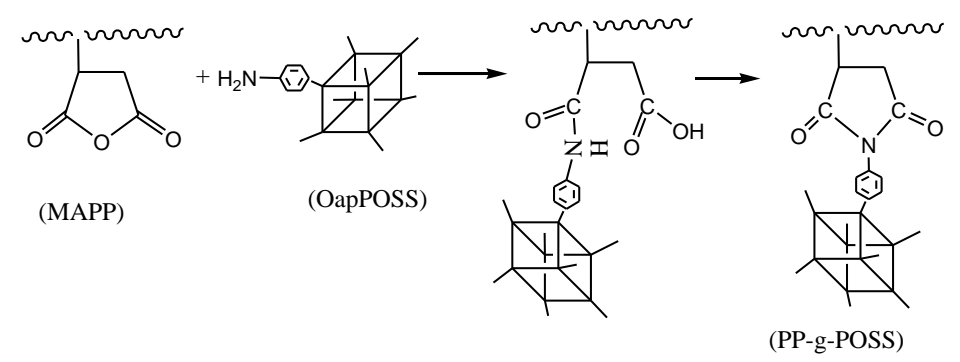

Scheme 1. Reaction of OapPOSS and MAPP.

The nanoparticles have two competing effects on the crystallization of polymers: inducing the nucleation and retarding the mobility of polymer chains. It is widely reported that nano clay could act as nucleating agents or retard crystallization of polymers which depended on the clay type, content, degree of exfoliation etc [33, 34]. Zheng et al. [35] reported that the ZnO nanoparticles would retard crystallization of polyamide 6 because the polymer chain mobility decreased due to the interactions between the polymer and $\mathrm{ZnO}$ filler. Weon et al. [36] reported $\mathrm{CaCO}_{3}$ particle would retard crystallization of high-crystallinity PP.
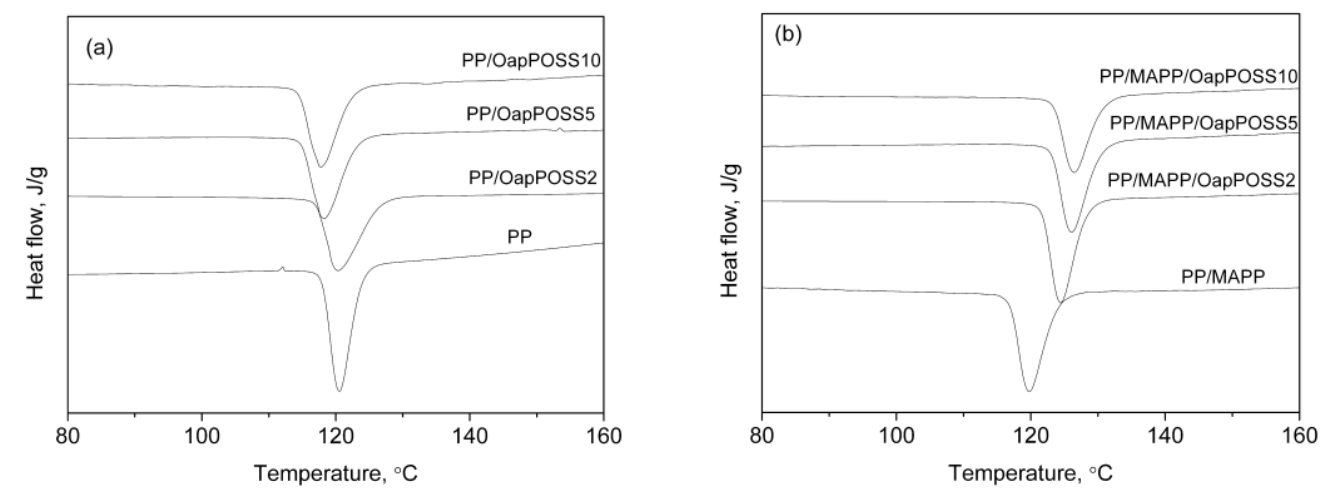

Fig. 4. Nonisothermal crystallization curves of PP composites: (a) PP/OapPOSS and (b) PP/MAPP/OapPOSS.
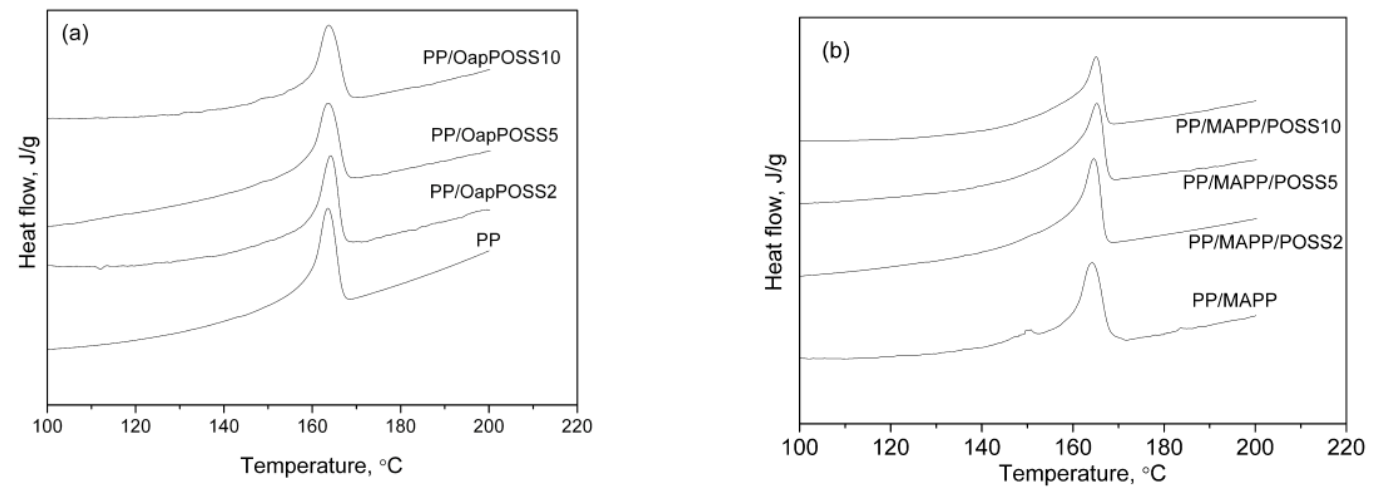

Fig. 5. Reheating melting curves of PP composites: (a) PP/OapPOSS and (b) PP/MAPP/OapPOSS. 
It is also reported that aggregated POSS crystals could act as nucleating agents for PP while dispersed POSS molecules decrease crystal growth ability [5, 25, 27]. Therefore, it can be speculated that the decrease of $T_{c}$ and degree of crystallinity is probably due to the fact that mobility of the PP chains is retarded by OapPOSS. While the OapPOSS particle become smaller and dispersed more uniformly when the OapPOSS reacted with MAPP after MAPP was added, the strong nucleation effect of the PP grafted OapPOSS would compensate and overlap the retardation effect. It is also reported by Jiang [37] and Cui [38] et al. that dynamically cured epoxy or Novolac resin have more effective nucleating effect when MAPP was added to PP/epoxy or PP/Novolac blends.

In all cases, the melting temperature $\left(T_{m}\right)$ of PP/MAPP/OapPOSS shifts to higher temperature compared with that of the corresponding PP and PP/OapPOSS. The higher $T_{m}$ values which mean the existence of more perfect and stable crystals of PP are probably due to the nucleating effect of OapPOSS grafted PP when MAPP was added.

The degree of crystallinity $\left(X_{c}\right)$ of PP can be calculated according to the following equations:

$X_{c}=\frac{\Delta H_{m}}{w \Delta H_{m}^{0}} \times 100 \%$

where $w$ is the weight fraction of the PP, $\Delta H_{m}$ is the enthalpy of fusion of the PP or the blends, $\Delta H_{m}^{0}(209 \mathrm{~J} / \mathrm{g})$ [39] denotes the melting enthalpy of perfect PP crystals. The degree of crystallinity of PP was calculated from the equation (1) and the data are listed in Table 1. It can be seen that the degree of crystallinity decreased in PP/OapPOSS. While the degree of crystallinity increased in PP/MAPP/OapPOSS at low OapPOSS content and decreased at $10 \%$ OapPOSS content.

Tab. 1. The nonisothermal crystallization parameters of PP composites.

\begin{tabular}{lccccc}
\hline \multicolumn{1}{c}{ Samples } & $T_{c},\left({ }^{\circ} \mathrm{C}\right)$ & $\begin{array}{c}T_{\text {onset }} \\
\left({ }^{\circ} \mathrm{C}\right)\end{array}$ & $\Delta H_{c}, \mathrm{~J} / \mathrm{g}$ & $X_{c,}(\%)$ & $T_{m},\left({ }^{\circ} \mathrm{C}\right)$ \\
\hline Contract PP & 120.5 & 123.9 & 64.9 & 31.1 & 163.6 \\
PP/OapPOSS2 & 120.4 & 126.7 & 57.1 & 27.9 & 164.3 \\
PP/OapPOSS5 & 118.3 & 123.1 & 52.9 & 26.6 & 163.8 \\
PP/OapPOSS10 & 117.8 & 122.6 & 54.2 & 28.8 & 163.9 \\
PP/MAPP & 119.7 & 124.1 & 64.2 & 30.7 & 164.4 \\
PP/MAPP/OapPOSS2 & 124.5 & 128.4 & 68.8 & 33.6 & 164.7 \\
PP/MAPP/OapPOSS5 & 126.1 & 130.4 & 64.7 & 32.6 & 165.3 \\
PP/MAPP/OapPOSS10 & 126.5 & 130.8 & 49.2 & 28.3 & 165.2 \\
\hline
\end{tabular}

The crystallization behavior of PP composites will be further discussed by POM analysis. Figure 6 shows POM micrographs of PP and PP composites that have been isothermal crystallized at $140{ }^{\circ} \mathrm{C}$ for $20 \mathrm{~min}$. As shown in Figure 6(a), the spherulites of PP are about $80 \mu \mathrm{m}$, the spherulites perfectly grow with the maltese cross clearly observed. The spherulite size of PP/OapPOSS5 is about $50 \mu \mathrm{m}$ due to the increase of heterogeneous nuclei when OapPOSS was added during blending. POSS increased the amount of heterogeneous nuclei in the PP matrix and decreased the spherulite size of PP. For the PP/MAPP/OapPOSS5, the spherulite 
size was further decreased and some spherulites were too small to be seen in the POM micrographs. It suggested that the reaction of MAPP with OapPOSS decreased the OapPOSS particle size while increased the particle number, which increased the number of PP spherulites and reduced the spherulite size.
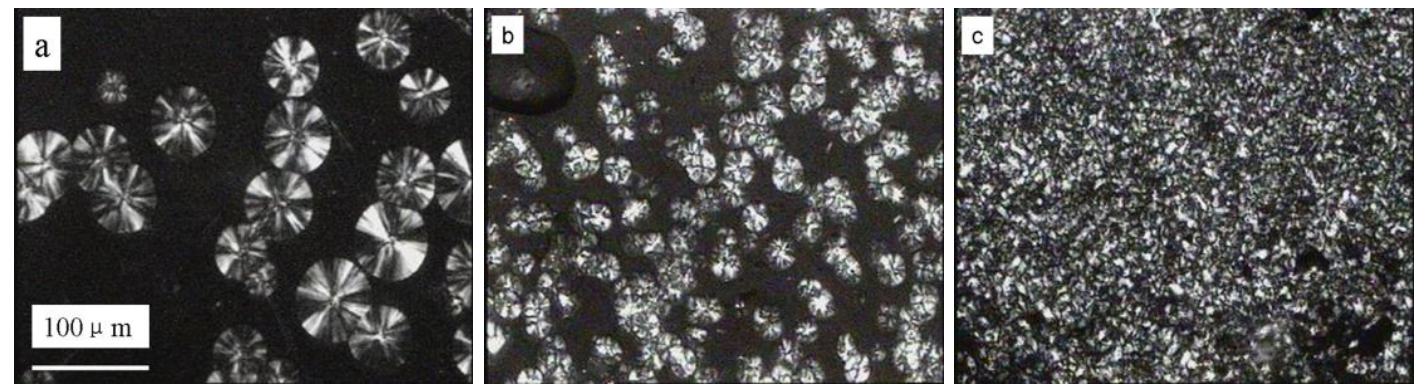

Fig. 6. Morphology of crystallization by POM (×200) (a) PP, (b) PP/OapPOSS5, (c) PP/MAPP/OapPOSS5.
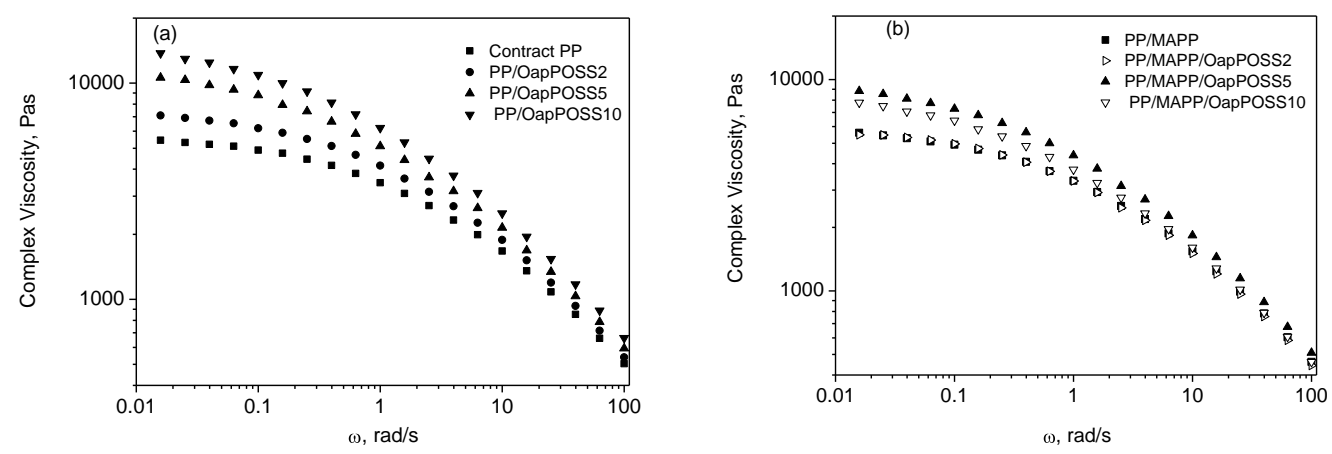

Fig. 7. Complex viscosity versus frequency for PP and PP composites: (a) PP/OapPOSS and (b) PP/MAPP/OapPOSS.
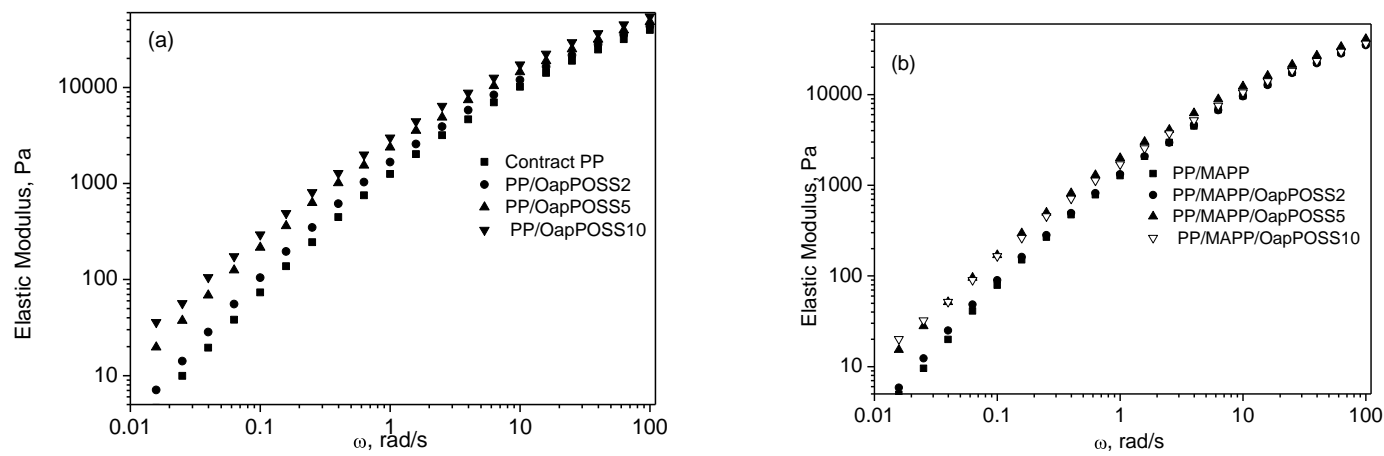

Fig. 8. Elastic modulus versus frequency for PP and PP composites: (a) PP/OapPOSS and (b) PP/MAPP/OapPOSS.

Figures 7, 8 and 9 show double logarithmic plot of the complex viscosity $\eta^{*}$, elastic modulus $G^{\prime}$ and viscous modulus $G$ " as functions of angular frequency $\omega$ for PP composites at $190{ }^{\circ} \mathrm{C}$ respectively. As shown in Figure 7, all curves present an evident shear thinning behavior with increasing frequency, indicating the pseudoplastic nature of PP. For PP/OapPOSS, $\eta^{*}$ increased with increasing 
OapPOSS content, while the $\eta^{*}$ of all the PP/MAPP/OapPOSS is lower than that of PP/OapPOSS at a same OapPOSS content. There are similar trend in elastic modulus $G^{\prime}$ and viscous modulus $G^{\prime \prime}$ for PP composites just as shown in Figures 8 and 9. The precise reasons for the difference in rheological behavior are not understood yet, but it is believed that this may be caused by the difference in OapPOSS particle size and the interaction between PP and OapPOSS when MAPP was added.
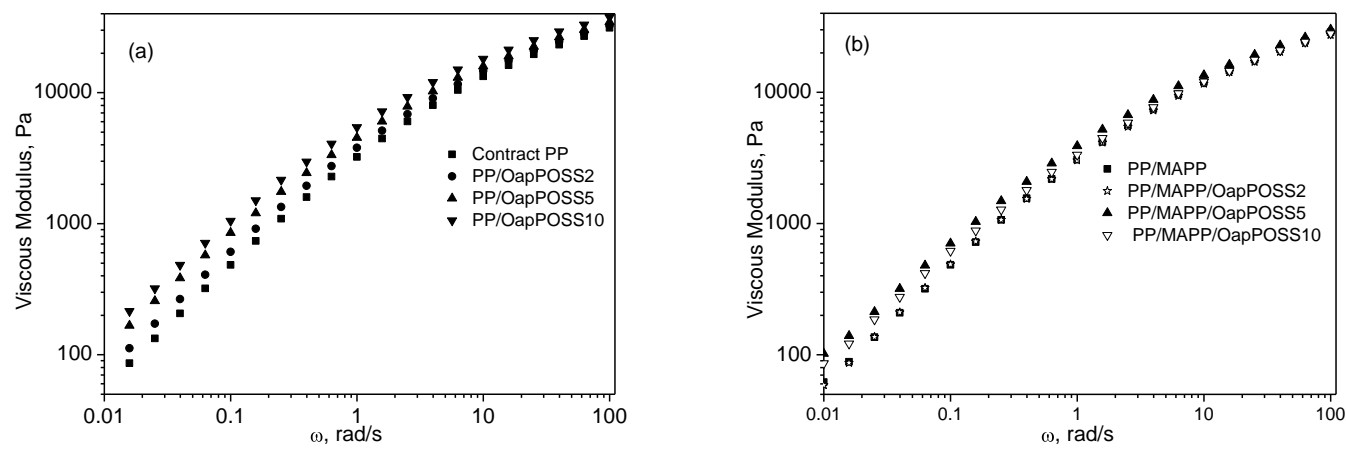

Fig. 9. Viscous modulus versus frequency for PP and PP composites: (a) PP/OapPOSS and (b) PP/MAPP/OapPOSS

Many factors can affect the rheological properties of polymer composites. Bomal [40] and Wang [41] found that surface treatment of calcium carbonate would decrease the surface energy and the particle-particle interactions, resulting in major viscosity reductions and decrease in the "apparent yield" value. OapPOSS would be encapsulated by PP chains and thus the particle-particle interaction decrease due to the reaction of OapPOSS and MAPP. On the other hand, the viscosity of polymer melts depends on the interlayer interaction. Interlayer slip or inhomogeneous flow would decrease the viscosity of polymer [42]. Inn et al. [43] have also reported that the interfacial tension would change and stress induced interfacial slippage may occur with the surface treatment of particles. Therefore, we can speculate that the easier interfacial slippage due to the larger OapPOSS particle numbers and small particle size after the addition of MAPP than that without MAPP could also induce the decreasing of viscosity of PP/MAPP/OapPOSS.

As shown in Figure $7, \eta^{*}$ of PP/MAPP/OapPOSS reached its maximum at $5 \%$ OapPOSS content. Xie et al. [44] found the viscosity of poly(vinyl chloride) (PVC) $/ \mathrm{CaCO}_{3}$ nanocomposite reached maximum at $2.5 \mathrm{wt} \% \mathrm{CaCO}_{3}$ and proposed that the spherical nanoparticles serve as "ball bearings", reducing the interlayer interaction of PVC melts and thereby decreasing the viscosity of $\mathrm{PVC} / \mathrm{CaCO}_{3}$ composite. We would also propose that the OapPOSS of PP/MAPP/OapPOSS serve as "ball bearing", reducing the interlayer interaction of PP melt and thereby decreasing the viscosity of PP/MAPP/OapPOSS composites. It is expected that the ball bearing become more prominent with increasing OapPOSS content and OapPOSS was encapsulated by PP chain when OapPOSS was reacted with MAPP.

The apparent shear viscosity $\eta$ versus apparent shear $\gamma$ of PP and PP composites is shown in Figure 10. All the materials show a shear thinning behavior and the value of 
viscosity decreases with increasing shear rate. The bilogarithmic plots are based on the Power Law model:

$\eta=k \gamma^{n-1}$ hence $\log \eta=\log k+(n-1) \log \gamma$

where $k$ is the consistency index and $n$ the power law index.
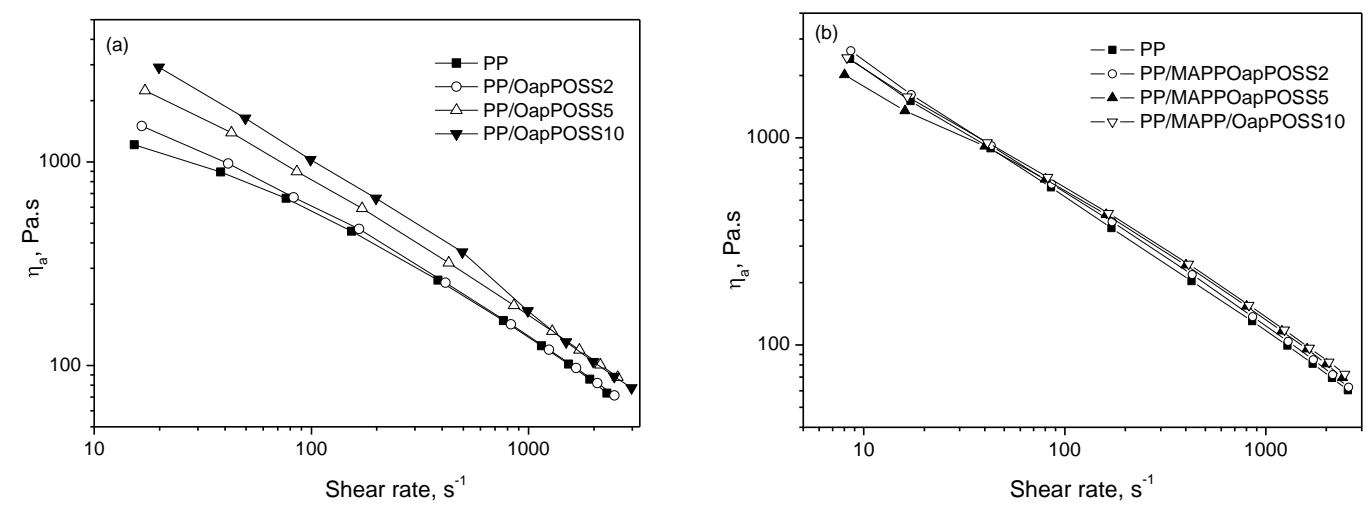

Fig. 10. Capillary rheological curves for PP composites: (a) PP/OapPOSS and (b) PP/MAPP/OapPOSS.

Tab. 2. Power law index $n$ of PP composites.

\begin{tabular}{lcccc}
\hline $\begin{array}{l}\text { QapPOSS content, } \\
\%\end{array}$ & 0 & 2 & 5 & 10 \\
\cline { 1 - 4 } PP/OapPOSS & 0.42 & 0.38 & 0.36 & 0.27 \\
PP/MAPP/OapPOSS & 0.36 & 0.35 & 0.41 & 0.39 \\
\hline
\end{tabular}

Figure 10 show that all the materials follow a power law relationship at low apparent shear rate. The value of $\eta$ at the same shear rate increased with increasing OapPOSS content for PP/OapPOSS, while little change happened to PP/MAPP/OapPOSS. Table 2 lists the power law indexes $(n)$ of PP composites. The $n$ is gradually decreased with increasing OapPOSS content for PP/OapPOSS and little change occured for PP/MAPP/OapPOSS. It is consistent with that of oscillatory rheological behavior. It can also be speculated that lower particle-particle interaction and greater "ball bearing" effect due to the reaction of OapPOSS and MAPP induce lower viscosity of PP/MAPP/OapPOSS than that of PP/OapPOSS.

\section{Conclusions}

Octaaminophenyl POSS (OapPOSS) was prepared as nanofiller and filled to isotactic PP. Maleic anhydride-grafted PP (MAPP) was selected as the compatibilizer. The morphology, crystallization and rheological behavior were strongly influenced when the MAPP was added. SEM showed that the MAPP could improve the compatibility between PP and OapPOSS and promote a fine dispersion of OapPOSS particles in PP matrix due to the reaction of amine groups of OapPOSS with maleic anhtydride groups of MAPP. DSC and POM analysis showed that OapPOSS retarded the crystallization of PP in PP/OapPOSS, while had a nucleating effect when MAPP was 
added. This is probably due to the combination of retardation and nucleation effects of OapPOSS to PP. The nucleation effect of the PP grafted OapPOSS would compensate and overlap the retardation effect when MAPP was added. Rheological analysis showed MAPP could decrease the viscosity of PP/OapPOSS probably due to the weak particle-particle interaction and interface slipping.

\section{Experimental part}

PP, F401 was produced by Liaoning Panjin Petrochemical Co., Ltd. China, with a melt flow index (MFI) of $2.3 \mathrm{~g} / 10 \mathrm{~min}\left(230^{\circ} \mathrm{C}, 2.16 \mathrm{~kg}\right)$. MAPP with a MAH content of $1 \mathrm{wt} \%$ was prepared by Shanghai Sunny New Technology Development Co., Ltd. (China).The OctaPhenyl POSS, MS0408 was purchased from Hybrid Plastic Co., USA. Octaaminophenyl POSS (OapPOSS) was synthesized according to following literature methods [18, 21].

The nitration of phenyl POSS was employed to prepare nitrophenyl POSS (OnpPOSS). $50 \mathrm{~g}$ of octaphenyl POSS (48.4 mmol) was slowly added to $300 \mathrm{ml}$ of fuming nitric acid with stirring at $0{ }^{\circ} \mathrm{C}$. After addition was completed, the solution was stirred for additional $30 \mathrm{~min}$ and then at room temperature for $20 \mathrm{~h}$. After filtration through glass wool, the solution was poured into $500 \mathrm{~g}$ of ice. A faintly yellow precipitate was collected by filtration and washed with $5 \mathrm{wt} \%$ of sodium carbonate solution and water to attain the value of $\mathrm{PH}$ 7. The obtained powder was dried to yield $59.7 \mathrm{~g}(42.8 \mathrm{mmol}, 88.5 \%)$ of samples. FTIR $\left(\mathrm{cm}^{-1}\right)$ with $\mathrm{KBr}$ powder: 1348 $(\checkmark N=0), 1530(v N=0), 1270-975(v \mathrm{Si}-\mathrm{O}-\mathrm{Si}) ;{ }^{1} \mathrm{H}$ NMR (acetone-d6, ppm): $8.7(\mathrm{t}$, 1.0H), 8.5 8.0 (m, 4.0H), $7.8(\mathrm{~m}, 2.8 \mathrm{H}) ;{ }^{13} \mathrm{C}$ NMR (acetone-d6, ppm): 153.3, 148.2, $140.3,138.0,134.7,133.7,131.6,131.6,131.5,130.2,129.3,128.8,128.2,125.7$, 125.2, 124.2, .

The reduction of OnpPOSS was employed to prepare OapPOSS. Typically, $15 \mathrm{~g}$ $\left(10.74 \mathrm{mmol},-\mathrm{NO}_{2}, 86.1 \mathrm{mmol}\right)$ of OnpPOSS and $10 \mathrm{wt} \% \mathrm{Pd} / \mathrm{C}(0.915 \mathrm{~g}, 0.861$ mmol) catalyst were charged into a $500 \mathrm{ml}$ flask equipped with a condenser and a magnetic stirrer under $\mathrm{N}_{2}$. Purified tetrahydrofuran (THF) $\left(\begin{array}{lll}120 & \mathrm{ml}\end{array}\right)$ and triethyleneamine $(120 \mathrm{ml}, 0.861 \mathrm{~mol})$ were added. The mixture was heated up to 60 ${ }^{0} \mathrm{C}$ and formic acid $(15.6 \mathrm{ml}, 0.345 \mathrm{~mol})$ was slowly dropped into the system. It was observed that gas $\left(\mathrm{CO}_{2}\right)$ was produced and the solution was gradually converted into two layers with the reaction proceeding. After $5 \mathrm{~h}, 75 \mathrm{ml}$ of water and $75 \mathrm{ml}$ of THF were added to the system until the slurry formed a black suspension. The $\mathrm{Pd} / \mathrm{C}$ catalyst was separated by filtration. The recovered catalyst was washed with N,N'dimethylformamide (DMF) for several times. All the solutions were combined into a flask and the solvents were removed by means of a rotary evaporator. To remove the residual solvent, the product was further dried in vacuo for 2 weeks at $60{ }^{\circ} \mathrm{C}$. The product was collected. Yield: $10.48 \mathrm{~g}(9.08 \mathrm{mmol}, 84.3 \%)$. FTIR $\left(\mathrm{cm}^{-1}\right)$ with $\mathrm{KBr}$ powder: 3365 ( $(\mathrm{N}-\mathrm{H}), 1120$ ( $(\mathrm{SSi}-\mathrm{O}-\mathrm{Si}) ;{ }^{1} \mathrm{H}$ NMR (DMSO-d6, ppm): 7.7-6.2 (b, 4.0H), $5.1 \sim 3.5$ (b, 2.0H); ${ }^{13} \mathrm{C}$ NMR (DMSO-d6, ppm): 154.0, 148.0, 136.4, 132.5, 129.5, 123.2, 120.2, 117.2, 115.4 .

Before blending, PP, MAPP and OapPOSS were dried at $60{ }^{\circ} \mathrm{C}$ under vacuum for about $12 \mathrm{~h}$. PP, OapPOSS and MAPP (10wt\% according to the total weight as if it was added) composites were prepared in the mixing chamber of a Haake Rheometer RC90 at $190{ }^{\circ} \mathrm{C}$ and $60 \mathrm{rpm}$ for $8 \mathrm{~min}$. The composition was moved out and compression molded in a press at $190{ }^{\circ} \mathrm{C}$ for $20 \mathrm{~min}$, then cold pressed to get samples for testing. 
The morphology of PP/POSS composites was observed by field emission scanning electron microscopy (SEM) (JSM-7401F, JEOL Co., Japan). The samples were fractured under cryogenic condition using liquid nitrogen. The fracture surfaces were coated with thin layers of gold of about $10 \mathrm{~nm}$.

The Fourier transform infrared spectroscopy (FTIR) was obtained using an FTIR spectrometer (Paragon 1000 from Perkin-Elmer Co., USA). The scan range was from 4400 to $400 \mathrm{~cm}^{-1}$ with a resolution of $4 \mathrm{~cm}^{-1}$. The composites were resolved in refluxing xylene, precipitated and washed by acetone, the precipitate was resolved and precipitated at least three times and dried at $80{ }^{\circ} \mathrm{C}$ for $12 \mathrm{~h}$. Samples of the precipitations were pressed to thin films for testing.

Thermal properties of samples were measured using a Perkin-Elmer DSC. All tests were performed in a nitrogen atmosphere with a sample weight of about $4 \mathrm{mg}$. All samples were first heated to $200{ }^{\circ} \mathrm{C}$ at $20^{\circ} \mathrm{C} / \mathrm{min}$ and kept for $3 \mathrm{~min}$ to eliminate prior thermal history. The specimen was subsequently cooled down to $25^{\circ} \mathrm{C}$ at a cooling rate of $10{ }^{\circ} \mathrm{C} / \mathrm{min}$ and then heated to $200{ }^{\circ} \mathrm{C}$ at $10{ }^{\circ} \mathrm{C} / \mathrm{min}$.

The crystallization of PP was studied on thin films by using an optical polarizing microscope LEICA-DMLPI, with an automatic hot-stage thermal control. Samples were sandwiched between microscope cover glasses, melted at $230{ }^{\circ} \mathrm{C}$ for 3 min and then rapidly cooled to $140{ }^{\circ} \mathrm{C}$ for isothermal crystallization for $30 \mathrm{~min}$. The PP spherulites were observed in micrographs taken at appropriate intervals.

Oscillatory rheological measurements were performed using a rotational rheometer (Gemini 200HR, Bohlin Co., UK) equipped with a parallel plate geometry using 25 $\mathrm{mm}$ diameter plates at $190{ }^{\circ} \mathrm{C}$ and frequency range from 0.01 to $100 \mathrm{rad} \cdot \mathrm{s}^{-1}$. Elastic modulus $\left(G^{\prime}\right)$, viscous modulus $\left(G^{\prime \prime}\right)$, complex viscosity $\eta^{*}$ were measured in the frequency sweep experiments.

Melt viscosity was measured using an Instron 4467 capillary rheometer (Instron Corp., USA) at $200^{\circ} \mathrm{C}$. The capillary die had a length (L) to diameter (D) ratio of $40 / 1$ and an entrance angle of $180^{\circ}$. The Rabinowitch and Bagley corrections were applied to all the experimental data.

\section{Acknowledgements}

The financial support from the National Natural Science Foundation of China (Project No 50603013) is acknowledged. The partial support from Shanghai Leading Academic Discipline Project (Project No P1502) is also acknowledged.

\section{References}

[1] Feher, F. J.; Newman, D. A.; Walzer, J. F. J. Am. Chem. Soc. 1989, 111, 1741.

[2] Zheng, L.; Farris, R. J.; Coughlin, E. B. Macromolecules 2001, 34, 8034.

[3] Waddon, A. J.; Zheng, L.; Farris, R. J.; Coughlin, E. B. Nano. Lett. 2002, 3, 1149.

[4] Zheng, L.; Waddon, A. J.; Farris, R. J.; Coughlin, E. B. Macromolecules 2002, 35, 2375.

[5] Fina, A.; Tabuani, D.; Frache, A.; Camino, G. Polymer 2005, 46, 7855.

[6] Chen, J. H.; Chiou, Y. D. J. Polym. Sci, Part B: Polym Phys 2006, 44, 2122.

[7] Fina, A.; Abbenhuis, H. C. L.; Tabuani, D.; Camino, G. Polym. Degrad. Stab. 2006, 9, 2275.

[8] Joshi, M.; Butola, B. S.; Simon, G.; Kukaleva, N. Macromolecules, 2006, 39, 1839 
[9] Baldi, F.; Bignotti, F.; Fina, A.; Tabuani, D.; Ricco, T. J.Appl.Poly.Sci. 2007, 105, 935.

[10] Chen, J. H.; Yao, B. X.; Su, W. B.; Yang, Y. B. Polymer, 2007, 48,1756.

[11] Haddad, T. S.; Viers, B. D.; Phillips, S. H. J.Inorg.Organomet.Polym. 2001, 11, 155.

[12] Costa, R. O. R.; Vasconcelos, W. L.; Tamaki, R.; Laine, R. M. Macromolecules, 2001, 34, 5398.

[13] Zhao, Y.; Schiraldi, D. A. Polymer, 2005, 46, 11640.

[14] Yoon, K. H.; Polk, M. B.; Park, J. H.; Min, B. G.; Schiraldi, D. A. Polym. Int. 2005, $54,47$.

[15] Ciolacu, F. C. L.; Choudhury, N. R.; Dutta, N.; Kosior, E. Macromolecules 2007, 40, 265.

[16] Ricco, L.; Russo, S.; Monticelli, O.; Bordo, A.; Bellucci, F. Polymer, 2005, 46, 6810.

[17] Baldi, F.; Bignotti, F.; Ricco, L.; Monticelli, O.; Ricco, T. J. Appl. Polym.Sci. 2006, 100, 3409.

[18] Ni, Y.; Zheng, S.; Nie, K. Polymer 2004, 45, 5557.

[19] Liu, Y.; Zheng, S.; Nie, K. Polymer 2005, 46, 12016.

[20] Zhang, Y.; Lee, S.; Yoonessi, M.; Liang, K.; Pittman, C. U. Polymer 2006, 47, 2984.

[21] Tamaki, R.; Tanaka, Y.; Asuncion, M. Z.; Choi, J.; Laine, R. M., J. Am. Chem. Soc. 2001, 123, 12416.

[22] Li, G.; Wang, L.; Ni, H.; Pittman Jr, C. U. J.Inorg. Organomet.Polym. 2001, 11, 123.

[23] Lee, Y. J.; Kuo, S. W.; Huang, W. J.; Lee, H. Y.; Chang, F. C. J. Polym. Sci., Part B: Polym. Phys. 2004, 42, 1127.

[24] Kuo, S. W.; Lin, H. C.; Huang, W. U. J.; Huang, C. F.; Chang, F. C. J. Polym. Sci. Part B: Polym. Phys.2006, 44673.

[25] Fu, B. X.; Yang, L.; Somani, R. H.; Zong, S. X.; Hsiao, B. S.; Phillips, S.; Blanski, R.; Ruth, P. J. Polym. Sci. Part B: Polym. Phys. 2001, 39, (22), 2727.

[26] Fina, A.; Abbenhuis, H. C. L.; Tabuani, D.; Frache, A.; Camino, G. Polym.Degrad.Stab. 2006, 91, 1064.

[27] Pracella, M.; Chionna, D.; Fina, A.; Tabuani, D.; Frache, A.; Camino, G. Macromol. Symp. 2006, 234, 59.

[28] Misra, R.; Fu, B. X.; Morgan, S. E. J. Polym. Sci. Part B: Polym. Phys. 2007, 45, 2441.

[29] Takala, M.; Karttunen, M.; Salovaara, P.; Kortet, S.; Kannus, K. IEEE T. Dielect. El. In. 2008, 15, 40.

[30] Zhou, Z. Y.; Zhang, Y.; Zhang, Y. X.; Yin, N. W. J. Polym. Sci., Part B: Polym. Phys. 2008, 46, 526.

[31] Liang, K.; Toghiani, H.; Guizhi, L. I.; Pittman Jr, C. U. J. Polym. Sci. Part A: Polym. Chem. 2005, 43, 3887.

[32] Zhang, Y.; Lee, S. H.; Yoonessi, M.; Toghiani, H.; Pittman Jr, C. U. J. Inorg. Organomet. Polym. Mater. 2007, 17,159

[33] Homminga, D.; Goderis, B.; Dolbnya, I.; Reynaers, H.; Groeninckx, G. Polymer, 2005, 46, 11359.

[34] Homminga, D. S.; Goderis, B.; Mathot, V. B. F.; Groeninckx, G. Polymer 2006, $47,1630$.

[35] Zheng, J.; Siegel, R. W.; Toney, C. G. J. Polym. Sci. Part B: Polym. Phys. 2003, 41, 1033. 
[36] Weon, J. I.; Sue, H. J. J. Mater. Sci. 2006, 41, 2291.

[37] Jiang, X.; Zhang, Y.; Zhang, Y. J. Polym. Sci. Part B: Polym. Phys. 2004, 42, 1181.

[38] Cui, L.; Zhang, Y.; Zhang, Y. J. Polym. Sci. Part B: Polym. Phys. 2006, 44, 3288.

[39] Tjong, S. C.; Xu, S. A. Polym. Int. 1997, 44, 95.

[40] Bomal, Y.; Godard, P. Polym. Eng. Sci. 1996, 36, 237.

[41] Wang, Y.; Wang, J. J. Polym. Eng.Sci.1999, 39,190.

[42] Mackay, M. E.; Henson, D. J. J.Rheo.1998, 42, 1505.

[43] Inn, Y.W.; Wang, S.Q. Langmuir, 1995, 11, 1589.

[44] Xie, X. L.; Liu, Q. X.; Li, R. K. Y.; Zhou, X. P.; Zhang, Q. X.; Yu, Z. Z.; Mai, Y. W. Polymer, 2004, 45,6665. 